\section{How to Produce Posters Using PowerPoint}

John J. Bozzola

Southern Illinois University

bozzola@siu.edu

Large format posters for scientific meetings can be produced using a variety of high-end layout programs. Microsoft PowerPoint (PP), although primarily used for producing digital slides for projection, can also be used to produce professional quality posters. It is easy to incorporate previously produced PP slides, Excel graphs, or word processor text into a PP poster. Our facility uses Macintosh computers, but a similar procedure should work with a PC platform. A detailed article will be written in a future issue.

1. Allocate as much memory to the program as possible $(100 \mathrm{MB}$ of RAM) to prevent crashes or lock-ups.

2. Generate a custom-sized document under File/Page, Setup/ Custom. The maximum allowable size in this program is 56 inches; however, the poster can be scaled up during printing. For example, if one needs a poster sized at $48 \times 96$ in, set up a page size of $24 \mathrm{H} \times 48 \mathrm{~W}$ and print at $200 \%$.

3. While still in Page Setup, click on the Options button to obtain the submenu associated with setting up the document for your printer. (In step 2, the document is set up for display or projection, whereas this step establishes the printing conditions.) While still in Options, select the target Printer, and then the remaining parameters, as follows: Page Attributes, Paper; Custom, Orientation; Portrait, Scale, $200 \%$ (in this example). Next, go to Custom Page Defaults and enter the dimensions of your current poster. At this point, although it may seem counterintuitive, the height and width set during the initial page setup in step 2 is reversed, i.e., Width: 24 , Height: 48 . (Note: this step may be necessary only if outputting to a PostScript printer or file such a PDF.) Assign a Custom Page Name and click the Add button to save the new page parameters. Return to Page Attributes and verify that your custom page $(48 \mathrm{H} \times 24 \mathrm{~W})$ is selected, and then click OK and to return to the Page Setup window. Click OK again to return to the desktop where your poster is being created.

4. Lay out the poster as usual with PP. Use at least 72 point font size for titles and 20-24 point for regular text. Avoid exotic fonts that may cause printing problems, but use standard sets such as Arial, Times, and Palatino. Text may be entered into standard text boxes in PP or copied and pasted from a word processing document. In the latter case, it may be necessary to reformat the text. Avoid auto-formatting, but instead enter indents and bullets manually. Images should be TIF format, 300 ppi, CMYK, or grayscale. Although other formats may work, PP handles TIF images more reliably. Since most posters are printed at $300 \mathrm{ppi}$, the images should be sized appropriately. For example, if an original image is $4 \times 5$ inches but will be scaled up $200 \%$, use 600 ppi since this will produce an $8 \times 10$ in. image with $300 \mathrm{ppi}$. Crop images to keep file sizes small. An Excel chart or figure can be inserted in your poster by using the Copy command in Excel and pasting it into the PP poster.

5. To use slides from a previous PP set, display the slides in the Slide Sorter, View/Slide Sorter. Position the mouse over the slide that is to be copied onto the poster and, while keeping the mouse button depressed, drag the slide onto the poster. After copying the necessary slides, arrange and re-size the individual slides as needed. If it is necessary to edit the slides, return to the original slide show and then move the edited slides onto the poster. Overly complicated slides with multiple levels and images may not transfer properly, so it may be necessary to simplify the slide or re-insert some parts of the graphics on the slides in your poster.

6. The most challenging part of poster production using PP is printing. Only the latest printer drivers and reliable connections should be used. It is strongly recommend that the PP poster is converted to a PDF (portable document format) using Adobe Acrobat as this provides an excellent print preview that can be proofed for spelling errors and graphic quality. In fact, printing converted PP posters using Adobe Acrobat has proven so reliable that this approach is utilized in our microscopy courses. The time necessary for working out the hardware and software settings is well spent since PP is so widely used.

\section{Microscopy $_{\text {AnD }}$ Microanalysis \\ Table of Contents Preview Volume 9, Number 2, April 2003}

\section{Editorial}

Charles Lyman

\section{Historical Bibliography}

- Key Events in the History of Electron Microscopy F. Haguenau, P.W. Hawkes, J.L. Hutchinson, B. SatiatJeunemaître, G.T. Simon, and D.B. Williams

Papers from the Australian Microbeam Analysis Society

- Monet's Painting under the Microscope Paula Dredge, Richard Wuhrer, and Matthew R. Phillips

- Cathodoluminescence Efficiency Dependence on Excitation Density in n-Type Gallium Nitride Matthew R. Phillips, Hagen Telg, Sergei O. Kucheyev, Olaf Gelhausen, and Milos Toth

\section{Application of Quantitative Electron Microscopy to the} Mineral Content of Insect Cuticle

Ron Rasch, Bronwen W. Cribb, John Barry, and

Christopher M. Palmer

- Charge-related Problems Associated with X-ray Microanalysis in the Variable Pressure Scanning Electron Microscope at Low Pressures

Brendan J. Griffin and Alesandra A. Suvorova

\section{News and Commentary}

\section{- Calendar of Meetings and Courses}

Indexed in Chemical Abstracts, Current Contents, and BIOSIS

MSA members receive both Microscopy Today and Microscopy and Microanalysis FREE! 